\title{
Prevalence of Atrial Fibrillation in patients attending emergency department of Shahid Gangalal National Heart Centre, Kathmandu, Nepal
}

\author{
Krishna Prasad Adhikari, ${ }^{1}$ Rabi Malla, ${ }^{2}$ Deepak Limbu, ${ }^{2}$ Binay Kumar Rauniyar, ${ }^{2}$ Sudhir Regmi, ${ }^{1}$ Anish \\ Hirachan, ${ }^{1}$ Yadav Bhatt ${ }^{2}$
}

\author{
${ }^{1}$ Department of cardiology, National Academy of Medical sciences, Bir Hospital, Kathmandu \\ ${ }^{2}$ Department of cardiology, Shahid Gangalal National Heart Centre, Bansbari, Kathmandu \\ Corresponding Author: Krishna Prasad Adhikari, National Academy of Medical sciences, Bir Hospital, Kathmandu, Nepal, \\ email: kpadhi01@yahoo.com
}

\begin{abstract}
Background and Aims: Atrial fibrillation (AF) is the most common sustained arrhythmia. This study aims to evaluate its prevalence in patients attending emergency department of tertiary care cardiac centre. Methods: It was a prospective observational study of 1012 consecutive patients who attended emergency department of Shahid Gangalal National Heart Centre from September 2014 to November 2014. Electrocardiogram, demographic features, diagnosis and clinical presentations were reviewed.

Results: Among the 1012 patients, 553 (54.6\%) were male and 459(45.4\%) were female. Mean age was $52.13 \pm 17$ years. A total of 140 patients $(13.8 \%)$ patients had AF. The mean age of patients with AF was 55 years. The prevalence of AF was higher in female than male (19.2\% Vs 9.4\%). Among the Rheumatic heart disease patients, seventy percentages of them had AF. Dyspnea was the commonest symptom of patients with AF followed by palpitation.

Conclusion: The prevalence of AF in our study is higher than in western world mainly because of endemic rheumatic heart disease.
\end{abstract}

Key words: Atrial fibrillation, hypertension, rheumatic heart disease, left venticular systole dysfunction

\section{Introduction}

Atrial fibrillation (AF), the most common sustained cardiac arrhythmia encountered in clinical practice, is increasing in prevalence. 1 The surface electrocardiogram characteristically demonstrates rapid atrial fibrillatory waves with changing morphology and rate and a ventricular response that is usually irregularly irregular. ${ }^{1}$ It is a potent risk factor for ischemic stroke, increasing the risk of stroke 5 -fold ${ }^{2}$ and accounting for approximately $15 \%$ of all strokes. Symptomatic AF may also reduce quality of life, functional status, and cardiac performance. ${ }^{3}$ The lifetime risk of developing AF is approximately $25 \%{ }^{4}$ The prevalence of $\mathrm{AF}$ increases substantially with age. ${ }^{5}$ The adjusted incidence and prevalence of $\mathrm{AF}$ is roughly double for each advancing decade of life, ${ }^{6,7}$ and, at any given age, men have an approximately $50 \%$ higher incidence of AF than women. ${ }^{6}$ Several previous studies have reported the prevalence of AF ranging from $1.2 \%$ to $2.8 \%$ in persons aged 60 through 69 years to $7.3 \%$ to $13.7 \%$ in persons aged 80 years or older. ${ }^{2,3,8,9}$ Among the various causes of AF, hypertension, rheumatic valvular heart disease, and congenital heart disease are the most commonly related conditions. ${ }^{10,11}$ Other causes include congestive heart failure, coronary artery disease, dilated, hypertrophic and restrictive cardiomyopathies and pulmonary hypertension.
There are no documented studies on prevalence of AF done in Nepal. Hence this study was conducted to evaluate the prevalence of $\mathrm{AF}$ in patients attending the emergency department of Shahid Gangalal National Heart Centre (SGNHC), Kathmandu, Nepal.

\section{Methods}

This was a single centre, prospective, observational study. Consecutive patients $(n=1012)$ who attended the emergency department of SGNHC from September 2014 to November 2014 were included in this study. The study was approved by institutional review board of National Academy of Medical Sciences, Kathmandu and SGNHC. Written informed consent was obtained from the patients.

An electrocardiogram of each patient was analysed independently by two observers and the differences were solved by consensus. The presence and absence of AF and the diagnosis of each patient were noted. The demographic features of all patients and presenting symptoms of patients with $\mathrm{AF}$ were also recorded. The statistical analysis was done with SPSS version 20. Categorical data were analysed by the Chi Square test. 
Descriptive statistics were computed and analysed as mean and standard deviation for continuous variables. p value less than 0.05 was taken as the level of significance at $95 \%$ confidence interval.

\section{Result}

A total of 1012 consecutive patients were evaluated. Among them $553(54.6 \%)$ were male and $459(45.4 \%)$ were female. The age of patients ranged from $10-97$ years with the mean age of $52.13 \pm 17.81$ years. Among the patients, 470 (46.4\%) had hypertension (HTN); 378 (37\%) patients had clinical or subclinical cardiac disease; 279 (27.6\%) had neither heart disease nor hypertension. Subclinical heart disease included patients with primary diagnosis of hypertension or non cardiac diagnosis with concomitant cardiac disease like asymptomatic left ventricular systolic dysfunction or mild to moderate mitral regurgitation on echocardiographic screening at the emergency department. Clinical cardiac disease includes patients with primary diagnosis of various cardiac diseases. Among 470 patients with primary diagnosis of hypertension, 115 (11.36\%) patients had subclinical cardiac disease. Patients with clinical and subclinical cardiac disease included 120 (11.8\%) patients with CAD, 74 (7.3\%) patients with rheumatic heart disease (RHD); 54 (5.3\%) patients with left ventricular systolic dysfunction, $36(3.5 \%)$ patients with non rheumatic mitral valve disease, $30(2.9 \%)$ patients with aortic valve disease; 24 (2.3\%) patients with cor pulmonale, 19 $(1.8 \%)$ patients who had undergone mitral valve replacement, 15 $(1.4 \%)$ patients with congenital heart disease and 3 patients each with constrictive pericarditis and post-permanent pacemaker insertion. A total of $104(10 \%)$ patients were diabetic.

Altogether $140(13.8 \%)$ patients had AF. The age of patients with $\mathrm{AF}$ ranged from $17-90$ years with the mean age of $56.06 \pm 18.08$ years. Out of them, $68(48.5 \%)$ had permanent AF, 48 (34\%) had persistent AF, 16 (11.4\%) had first time detected $\mathrm{AF}, 8(5.71 \%)$ had paroxysmal $\mathrm{AF}$. The prevalence of $\mathrm{AF}$ in various clinical conditions is given in Table 1 . The prevalence of AF was higher in patients with clinical and subclinical cardiac disease $(28 \%)$ as compared to those without hypertension and heart disease $(1.43 \%)$ which was statistically significant $(\mathrm{p}<0.05$; odds ratio $17.73,95 \%$ CI $7.6-41.05)$. AF was more prevalent among females than males $(19.2 \%$ vs $9.4 \%$; $<<0.05$; odds ratio 2.039; CI 1.48 - 2.80). Prevalence of AF in hypertensive patients was $5.95 \%$ which was significantly higher $(\mathrm{p}<0.05$; odds ratio 2.88 , CI 1.17 - 7.05) than in those without HTN (1.43\%). The study showed increasing prevalence of AF with age. The prevalence of $\mathrm{AF}$ above 60 years and between 10 and 60 years was $18 \%$ and $11.7 \%$, respectively $(\mathrm{p}=0.0087)$. Age distribution of atrial fibrillation is shown in Table 2. Table 3 shows the burden of $\mathrm{AF}$ in the various clinical conditions in the study population.

Table 1. Prevalence of AF $(\mathrm{n}=140)$

\begin{tabular}{lcc} 
Clinical Condition & $\%$ & P-value \\
Rheumatic heart disease & 70.27 & $<0.01$ \\
$\quad$ Mitral stenosis & 70.00 & $<0.01$ \\
\multicolumn{1}{c}{ Mitral regurgitation } & 66.66 & $<0.01$ \\
Mitral stenosis + regurgitation & 76.92 & $<0.01$ \\
Hypertension & 5.95 & 0.017 \\
LV systolic dysfunction & 27.77 & $<0.01$ \\
Post MVR & 68.42 & $<0.01$ \\
Non rheumatic mitral disease & 19.44 & $<0.01$ \\
Diabetes mellitus & 6.73 & 0.04 \\
COPD cor pulmonale & 16.66 & $<0.01$ \\
CAD & 3.33 & 0.49 \\
Non cardiac non HTN patients & 1.43 & 1.00 \\
Congenital heart disease & 20.00 & 0.01 \\
Aortic valve disease & 6.66 & 0.17 \\
Electrocution & &
\end{tabular}

Table 2 Prevalence of AF according to age group ( $n=140)$

\begin{tabular}{|llll}
\hline Age & Patient with $\mathbf{A F b}$ & Total patient & $\%$ \\
\hline $10-20$ & 4 & 32 & 11.11 \\
\hline $21-30$ & 12 & 89 & 11.88 \\
\hline $31-40$ & 16 & 138 & 10.38 \\
\hline $41-50$ & 24 & 168 & 12.50 \\
\hline $51-60$ & 24 & 172 & 12.24 \\
\hline $61-70$ & 23 & 122 & 15.86 \\
\hline $71-80$ & 27 & 109 & 19.85 \\
$>80$ & 10 & 42 & 19.23 \\
\hline
\end{tabular}


Table 3. Burden of AF according to diagnosis $(n=140)$

$\begin{array}{lc}\text { Diagnosis } & \text { AF } \mathrm{n}(\% \text { of total AF) } \\ & 52(37.14) \\ \text { Rheumatic heart disease } & 28(20.00) \\ \quad \text { Mitral stenosis } & 14(10.00) \\ \quad \text { Mitral regurgitation } & 10(7.14) \\ \quad \text { Mitral stenosis + regurgitation } & 28(20.00) \\ \text { Hypertension } & 15(10.71) \\ \text { LV systolic dysfunction } & 13(9.28) \\ \text { Post MVR } & 7(5.00) \\ \text { Non rheumatic mitral disease } & 7(5.00) \\ \text { Diabetes mellitus } & 4(2.85) \\ \text { COPD cor pulmonale } & 4(2.85) \\ \text { CAD } & 4(2.85) \\ \text { Non cardiac non HTN patients } & 3(2.14) \\ \text { Congenital heart disease } & 2(1.42) \\ \text { Aortic valve disease } & 1(0.71) \\ \text { Electrocution } & \end{array}$

The prevalence of AF in rheumatic mitral stenosis, rheumatic mitral regurgitation and combined rheumatic mitral stenosis and regurgitation were $70 \%, 66 \%$ and $76 \%$, respectively. There were 19 mitral valve replacement patients with $68 \%$ having AF. The prevalence of $\mathrm{AF}$ in patients with type 2 diabetes and coronary artery disease was found to be $7 \%(\mathrm{p}=0.046)$ and $3.3 \%(\mathrm{p}=0.49)$, respectively.

Among patients with $\mathrm{AF}$, the most common symptom observed on presentation was dyspnea 67 (48\%) followed by palpitations $46(33 \%)$, stroke/transient ischemic attack (10\%) and peripheral oedema (3\%). 16 percent patients presented with symptoms unrelated to AF. One of the patients was asymptomatic.

\section{Discussion}

$\mathrm{AF}$ is the most common sustained arrhythmia. There is a fivefold increased risk of stroke and three-fold increased risk of heart failure with AF, resulting in overall a higher morbidity and mortality ${ }^{12}$. This study evaluated the prevalence of atrial fibrillation in patients attending the emergency department of a tertiary cardiac care centre. The overall prevalence of $\mathrm{AF}$ in our study was $140(13.8 \%)$ patients.

Previous studies on prevalence of AF were of two basic types: population based and hospital based. The population based studies, used data from periodic checkups reflect general population. The first major population based studies were Western Australia Study, The Rochester Study ${ }^{13}$, The Framingham Study8, The Cardiovascular Health Study ${ }^{3}$. Based on these four studies, Feinberg et $\mathrm{al}^{5}$ reported the prevalence of $\mathrm{AF}$ to be $0.89 \%$ in United States in 1991 . The prevalence of AF in hospital based studies was higher than the population based studies. Among patients attending general hospital, the prevalence of AF was $2.5 \%$ in Japan ${ }^{14}$. The prevalence of AF was $10.4 \%$ in 1637 acutely ill patients admitted to Auckland Hospital in $1999 .{ }^{15}$ In the 50,000 consecutive patients of the Heart Station in Michael Reese Hospital ${ }^{16}$, the prevalence of AF was $11.7 \%$. The Hokkaido Atrial Fibrillation study $\operatorname{group}^{17}$ found the prevalence of $\mathrm{AF} 14 \%$ in 20,000 patients visiting cardiovascular clinics in 13 hospitals in Hokkaido, Japan in 2000.

The majority of previous studies have shown a higher prevalence of $\mathrm{AF}$ in males compared to females. The Framingham Heart Study showed that men have a 1.5 times greater risk of developing
$\mathrm{AF}$ than women ${ }^{6}$. In our study, $19.2 \%$ female had AF compared to $9.4 \%$ of males. This reflected a higher prevalence of RHD in females of $72 \%(n=61)$. Similar female dominance is seen in Indian Heart Rhythm Society Atrial Fibrillation (IHRS AF) registry with female occupied $51 \%$ of patients with $\mathrm{AF}^{18}$

The mean age of patients with AF in our study was $56.09+18.04$ years. In Indian cohort of REALIZE $\mathrm{AF}^{19}$ study and in the IHRS $\mathrm{AF}^{18}$ registry, the average age was 60 and 54 respectively. The average age of AF patients in Nepalese and Indian patients was a decade younger than the western studies. The mean age of patients with rheumatic valvular heart disease and $\mathrm{AF}$ was 40 years in our study. The mean age of patients with rheumatic valvular heart disease and AF in the CRAFT study 20 was 38 years.

RHD is endemic in Asia, Africa, South America and Middle East. ${ }^{21}$ Our study included 74 (7.3\%) patients of RHD with prevalence of $\mathrm{AF}$ of $70 \%$. AF in RHD contributed to $37.14 \%$ of total AF burden in our study. Echocardiographic evaluation of 137 consecutive patients with AF in a tertiary care centre from north India showed that $61.31 \%$ of $\mathrm{AF}$ was caused by RHD ${ }^{22}$. RHD, particularly with mitral valve disease, results in increased atrial chamber pressures, atrial stretch, increased atrial size, atrial muscle disruption, and fibrosis. Myocardial stretch slows conduction velocity, shortens refractory periods, increases the dispersion of refractoriness, and stimulates ectopic excitations, all lead to intra-atrial re-entry and fibrillation.

Left ventricular systolic dysfunction accounted for $54(5.3 \%)$ of total patients evaluated with $27.7 \%$ prevalence of AF. A population based study of people $>65$ years from the United States of America showed a prevalence of AF in patients with cardiovascular disease of $9.1 \%$ and in patients without cardiovascular disease of $1.6 \% .^{3} \mathrm{AF}$ occurs in about $10 \%$ of patients with NYHA class I or class II heart failure and in about $50 \%$ of patients with class IV.23

A total of $470(46.4 \%)$ patients in our study had HTN with $5.95 \%$ prevalence of AF. HTN was the underlying disease in $20 \%$ of patients with AF. In the Framingham heart study, HTN and diabetes increased the risk of AF by 1.5 fold.24 HTN was present in $59.1 \%$ of patients with AF in J-RHYTHM registry. ${ }^{25}$ The prevalence of $\mathrm{AF}$ in diabetes and $\mathrm{CAD}$ was $7 \%(\mathrm{p}=0.046)$ and $3.3 \%(p=0.49)$ respectively.

The present study is not free of limitations despite being the first prevalence study. It is relatively a small study. Being a hospital based study; it doesn't truly reflect the population prevalence.

\section{Conclusion}

The prevalence of AF was found to be high in a population with endemic RHD. Management of patients with AF to decrease morbidity and mortality is very important. Prevention strategies should focus on reducing RHD, appropriate treatment of HTN and left ventricular systolic dysfunction.

\section{References}

1. EN Prystowsky ,BJ Padanilam ,AL Waldo. Hurst's: The Heart. Eds. Fuster V, Walsh RA, Harrington RA et al. McGraw Hill, 13th Edition 2011: 963-81.

2. WB Kannel, PA Wolf, EJ Benzamin, et al. Prevalence, Incidence, Prognosis and predisposing condition for atrial fibrillation: population based estimates. Am J Cardiol.1998;82:2N-9N.

3. CD Furberg, BM Psaty, TA Manolio,et al. Prevalence of atrial fibrillation in elderly subjects (the Cardiovascular Health Study). Am J Cardiol. 1994;74:236-241. 
4. $\quad$ DM Lloyd-Jones ,TJ Wang, EP Leip, et al. Lifetime risk for development of atrial fibrillation: the Framingham Heart Study. Circulation. 2004;110: 1042-6.

5. WM Feinberg, JL Blackshear, A Laupacis et al. Prevalence, age distribution, and gender of patients with atrial fibrillation: analysis and implications. Arch Intern Med. 1995; 155:469473.

6. EJ Benjamin, D Levy, SM Vaziri et al. Independent risk factors for atrial fibrillation in a population-based cohort: the Framingham Heart Study. JAMA. 1994; 271: 840-4.

7. AS Go, EM Hylek, KA Phillips et al. Prevalence of diagnosed atrial fibrillation in adults: national implications for rhythm management and stroke prevention: the Anticoagulation and Risk Factors in Atrial Fibrillation (ATRIA) Study. JAMA. 2001;285:2370-5.

8. PA Wolf, RD Abbott,WB Kannel . Atrial fibrillation as an independent risk factor for stroke: the Framingham Study. Stroke. 1991;22:983-988.

9. FR Lake, KJ Cullen, NH de Klerk, et al. Atrial fibrillation and mortality in an elderly population. Aust $\mathrm{N} Z \mathrm{~J}$ Med. 1989;19:321-326.

10. GY Lip, DG Beevers. ABCs of atrial fibrillation: history, epidemiology and importance of atrial fibrillation. Br Med J. 1995;311: 1361-1363.

11. MA Allessie, PABoyden, AJ Camm , et al. Pathophysiology and prevention of atrial fibrillation. Circulation. 2001;103:769-777.

12. CraigT January, L Samuel Wann, Joseph S Alpert et al. ACC/AHA/HRS 2014 guidelines for the management of patients with atrial fibrillation. Circulation. 2014;129:000 000

13 SJ Phillips, JP Whisnant, WM O'fallon et al. Prevalence of cardiovascular disease and diabetes mellitus in residents of Rochester Minnoseta. Mayo Clin Proc. 1990;65:344-359
14 H Atarashi, T Fujiwara, H Saito et al. Frequency of cardiac arrhythmias and age related changes in general hospital report from Inada-Nobirito hospital in 1983.Jpn J Geriat 1986;23:41-49

15. FM Stewart, Y Singh, S Persson, et al. Atrial fibrillation: prevalence and management in an acute general medical unit. Aust NZ J Med. 1999;29:51-8

16. LN Katz, A Pick. Classification and frequency of arrhythmias. Clinical electrocardiography.Lea and Febiger, Philadelphia. 1956: 40-44

17. F Tomita, T Kohya, M Sakurai, et al. Prevalence and clinical characteristics of patients with atrial fibrillation: analysis of 20,000 cases in Japan. Jpn Circ J. 2000;64:653-8

18. IHRS AF registry. Presented in annual IHRS meeting 2012, Mumbai.

19. C. Narasimhan, JS Verma, AGR Kishore et al. The REALIZE AF registry, an international observational, cross sectional survey evaluating atrial fibriallation management and cardiovascular risk profile of AF patients - Indian subset data of the REALIZE AF study. Presented at the ISE meeting 2012,jaipur.

20. A Vora, D Karnad, V Goyal et al. Control of rate versus rhythm in rheumatic atrial fibrillation - a randomised study. Indian Heart Journal. 2004;56: 110 - 116

21. MD Seckeler, TR Hoke. The worldwide epidemiology of acute rheumatic fever and rheumatic heart disease. Clin Epidemiol.2011;3:67

22. Rajeev Bhardwaj. Atrial fibrillation in a tertiary care institute - a prospective study. Indian Heart J. 2012; 64(5): 476-478.

23. JR Ehrlich, S Nattel, SH Hohnloser. Atrial fibrillation and congestive heart failure: specific considerations at the intersection of two common and important cardiac disease sets. J Cardiovasc Electrophysiol. 2002; 13:399-405

24. WB Kannel. Epidemiology of atrial fibrillation: risk factors and hazards. www.fac.org.ar

25. H Atarashi, H Inoue, K Okumura et al. Present status of anticoagulation treatment in Japanese patients with atrial fibrillation- a report from the J-RHYTHM registry. Circ J. 2011;75:1328-33
Cite this article as: Krishna Prasad Adhikari, Rabi Malla,Deepak Limbu, et al. Prevalence of Atrial Fibrillation in patients attending emergency department of Shahid Gangalal National Heart Centre, Kathmandu, Nepal. Nepalese Heart Journal 2016; 13 (1): 1-4. 